\title{
A MULTIDIMENSIONAL GENERALIZATION OF ROE'S FLUX DIFFERENCE SPLITTER FOR THE EULER EQUATIONS
}

\author{
H. Deconinck, ${ }^{1}$ P. L. RoE ${ }^{2}$ and R. Struiss ${ }^{1}$ \\ 'von Karman Institute for Fluid Dynamics, Chaussée de Waterloo 72, 1640 Rhode-St-Genèse, Beigium \\ ${ }^{2}$ Department of Aerospace Engineering, University of Michigan, Ann Arbor, MI 48109, U.S.A.
}

(Received 1 April 1992; in revised form 8 September 1992)

\begin{abstract}
Upwind methods for the 1-D Euler equations, such as TVD schemes based on Roe's approximate Riemann solver, are reinterpreted as residual distribution schemes, assuming continuous piecewise linear space variation of the unknowns defined at the cell vertices. From this analysis three distinct steps are identified, each allowing for a multidimensional generalization without reference to dimensional splitting or 1-D Riemann problems. A key element is the necessity to have continuous piecewise linear variation of the unknowns, requiring linear triangles in two space dimensions and tetrahedra in three space dimensions. Flux differences naturally generalize to flux contour integrals over the triangles. Roe's flux difference splitter naturally generalizes to a multidimensional flux balance splitter if one assumes that the parameter vector variable is the primary dependent unknown having linear variation in space. Nonlinear positive and second-order scalar distribution schemes provide a true generalization of the TVD schemes in one space dimension. Although refinements and improvements are still possible for all these elements, computational examples show that these generalizations present a new framework for solving the multidimensional Euler equations.
\end{abstract}

\section{REVIEW OF ROE'S FLUX DIFFERENCE SPLITTER FOR THE 1-D EULER EQUATIONS}

We begin with a review of Roe's flux difference splitting (FDS) scheme for the 1-D Euler equations in a setting very close to the description given in Ref. [1]. In this formulation no explicit appeal is made to finite volumes nor to the Riemann problem as a building block for the solution, which turns out to be more suitable for a multidimensional generalization-as discussed in Section 2. Considering the system of Euler equations in one space dimension in conservation form $\mathbf{U}_{t}+\mathbf{F}_{x}=0$, with $\mathbf{U}$ and $\mathbf{F}$ the vectors of conserved variables and fluxes, three distinct steps can be recognized in formulating Roe's scheme.

\subsection{Wave decomposition step}

In this first step, which is trivial in 1-D, the flux divergence is decomposed into a number of scalar wave contributions. Starting from an eigenvector projection of the gradient of the conservative variable, the decomposition is given by

$$
\mathbf{U}_{x}=\sum_{k=1}^{3} \alpha^{k} \mathbf{r}^{k}, \quad \mathbf{F}_{x}=\sum_{k=1}^{3} \lambda^{k} \alpha^{k} \mathbf{r}^{k},
$$

where $\lambda^{k}(\mathbf{U})$ and $\mathbf{r}^{k}(\mathbf{U})$ are the eigenvalues and right eigenvectors of $A(\mathbf{U})=\mathbf{F}_{\mathbf{U}}$, the Jacobian matrix containing the derivatives of the flux vector with respect to the conservative variables. Hence, the decomposition models a general perturbation as a superposition of three simple wave contributions. The waves are traveling with speeds $\lambda \vec{\imath}_{x}$ along the $x$-axis, and have a strength $\alpha^{k}$ uniquely determined by inverting equation (1a), giving $\alpha^{k}=1^{k} U_{x}$, where $l^{k}$ are the left eigenvectors of $A$ such that $\mathbf{r}^{k} 1^{m}=\delta_{k m}$, with $\delta_{k m}$ the Kronecker symbol.

\subsection{Conservative linearization}

In the second step, a conservative linearization is constructed, which is in fact a discrete counterpart for equations $(1 \mathrm{a}, \mathrm{b})$, taking into account some requirements for conservation. For this, it is assumed that the solution is sought as a discrete mesh function $\left\{\mathbf{U}_{i}, i=1, \ldots, N\right\}$ on an irregularly spaced grid with meshpoints $x=x_{i}$. Precisely as in the finite element method using linear elements in space, the solution is represented as a continuous function, piecewise linear over the 
intervals $\left[x_{i}, x_{i+1}\right]$ with length $\Delta x_{i+\frac{1}{2}}=x_{i+1}-x_{i}$. Discrete gradients are defined in a consistent way as averages over a cell $\left[x_{i}, x_{i+1}\right]$, once a particular variable $\mathbf{Q}$ having linear variation is specified:

$$
\widehat{\mathbf{U}_{x}}=\frac{1}{\Delta x_{i+\frac{1}{2}}} \int_{x_{i}}^{x_{i+1}} \mathbf{U}_{x}(\mathbf{Q}) \mathrm{d} x, \quad \widehat{\mathbf{F}_{x}}=\frac{1}{\Delta x_{i+\frac{1}{2}}} \int_{x_{i}}^{x_{i+1}} \mathbf{F}_{x}(\mathbf{Q}) \mathrm{d} x .
$$

Straightforward integration of these expressions produces the flux difference and conservative variable difference over a cell. Alternatively, one may use the fact that $\mathbf{Q}_{x}$ is constant over the cell and write $\mathbf{U}_{x}=(\partial \mathrm{U} / \partial \mathbf{Q}) \mathbf{Q}_{x}$ and $\mathbf{F}_{x}=(\partial \mathbf{F} / \partial \mathbf{Q}) \mathbf{Q}_{x}$. Requiring that the integration using the chain rule is exact, and hence that the linearized expression is conservative, uniquely defines the linearized matrices as

$$
\frac{\overline{\partial \mathbf{U}}}{\partial \mathbf{Q}}=\frac{1}{\Delta x_{i+\frac{1}{2}}} \int_{x_{i}}^{x_{i}+1} \frac{\partial \mathbf{U}}{\partial \mathbf{Q}} \mathrm{d} x, \quad \frac{\bar{\partial} \mathbf{F}}{\partial \mathbf{Q}}=\frac{1}{\Delta x_{i+\frac{1}{2}}} \int_{x_{i}}^{x_{i}+1} \frac{\partial \mathbf{F}}{\partial \mathbf{Q}} \mathrm{d} x .
$$

The choice of the primary variable (e.g. the conservative, or some primitive variable) with linear variation is based solely on the ease of computing equations (3a, b) analytically. A particularly easy form is obtained, which is, moreover, identical to Roe's FDS scheme, if one selects for $\mathbf{Q}$ the parameter vector $Z=\left(z^{1}, z^{2}, z^{3}\right)^{\mathrm{T}}=\sqrt{\rho}(1, u, H)^{\mathrm{T}}$ as the variable with linear variation over a cell such that its gradient is constant over the cell, given by $\mathbf{Z}_{x}=\widehat{\mathbf{Z}_{x}}=\left(\mathbf{Z}_{i+1}-\mathbf{Z}_{i}\right) / \Delta x_{i+\frac{1}{2}}$. The reason for using $\mathbf{Z}$ as the primary unknown is precisely that each component of the conservative variable $U$ and the flux vector $F$ is a bilinear function of the components of $Z$, and as a consequence $\partial \mathbf{U} / \partial \mathbf{Z}=M_{\mathbf{Z}}$ and $\partial \mathbf{F} / \partial \mathbf{Z}=A_{\mathbf{Z}}$ are just linear functions in the components of $\mathbf{Z}$ [2]. Therefore, integration over the cell as needed in equations $(3 a, b)$ reduces to taking the arithmetic mean, giving

$$
\widehat{\mathbf{U}_{x}}=M_{Z}(\overline{\mathbf{Z}}) \widehat{\mathbf{Z}_{x}}, \quad \widehat{\mathbf{F}_{x}}=A_{Z}(\overline{\mathbf{Z}}) \widehat{\mathbf{Z}_{x}},
$$

where

$$
\overline{\mathbf{Z}}=\frac{\mathbf{Z}_{i}+\mathbf{Z}_{i+1}}{2}=\left(\begin{array}{c}
\frac{\left(\sqrt{\rho_{i}}+\sqrt{\rho_{i+1}}\right)}{2} \\
\frac{u_{i} \sqrt{\rho_{i}}+u_{i+1} \sqrt{\rho_{i+1}}}{2} \\
\frac{H_{i} \sqrt{\rho_{i}}+H_{i+1} \sqrt{\rho_{i+1}}}{2}
\end{array}\right) .
$$

As a result, the linearization $(4 a, b)$ is conservative in the sense that the gradients, multiplied with the length of the corresonding cell, telescope, i.e. their sum involves only contributions from meshpoints $x_{1}$ and $x_{N}$. Equations $(4 \mathrm{a}, \mathrm{b})$ give the linearization in terms of $\mathbf{Z}_{x}$; the linearization of the flux divergence in terms of $\mathbf{U}_{x}$ is simply obtained by inverting equation (4a) and substituting in equation (4b). Since the matrix $A_{Z}(\mathrm{Z}) M_{Z}^{-1}(\mathrm{Z})=(\partial \mathrm{F} / \partial \mathrm{U})(\mathrm{Z})=A(\mathrm{Z})$ is precisely the Jacobian matrix of the fluxes with respect to the conservative variable, the flux divergence can also be written as $\widehat{F}_{x}=A(\overline{\mathbf{Z}}) \hat{\mathbf{U}}_{x}$. This expression is easily recognized as the familiar expression for the flux difference over a cell in Roe's FDS. The conservative decomposition under the form of equations $(1 \mathrm{a}, \mathrm{b})$ is then easily found as

$$
\widehat{\mathbf{U}_{x}}=\frac{\mathbf{U}_{i+1}-\mathbf{U}_{i}}{\Delta x_{i+\frac{1}{2}}}=\sum_{k=1}^{3} \widehat{\alpha^{k}} \overline{\mathbf{F}}^{k}, \quad \widehat{\mathbf{F}_{x}}=\frac{\mathbf{F}_{i+1}-\mathbf{F}_{i}}{\Delta x_{i+\frac{1}{2}}}=\sum_{i=1}^{3}{\overline{\lambda^{k}}}_{\alpha^{k}} \overline{\mathbf{F}}^{k}
$$

The wave strength is again computed by inverting equation (6a), giving

$$
\widehat{\alpha^{k}}=\mathbb{T}^{\mathbf{k}} \widehat{\mathbf{U}_{x}}=\mathbf{T}^{\mathbf{k}} \frac{\mathbf{U}_{i+1}-\mathbf{U}_{i}}{\Delta x_{i+\frac{1}{2}}},
$$

while the linearized eigenvectors and eigenvalues are just the analytical expressions for the Jacobian $A$, evaluated in the state $\overline{\mathbf{z}}$. For example, in the state $\overline{\mathbf{z}}$, one has

$$
\vec{\rho}=\rho(\overline{\mathbf{Z}})=\left(\frac{\sqrt{\rho_{i}}+\sqrt{\rho_{i+1}}}{2}\right)^{2}, \quad \bar{u}=u(\overline{\mathbf{Z}})=\frac{\sqrt{\rho_{i} u_{i}}+\sqrt{\rho_{i+1}} u_{i+1}}{\sqrt{\rho_{i}}+\sqrt{\rho_{i+1}}},
$$




$$
H=H(\overline{\mathbf{Z}})=\frac{\sqrt{\rho_{i}} H_{i}+\sqrt{\rho_{i+1}} H_{i+1}}{\sqrt{\rho_{i}}+\sqrt{\rho_{i+1}}}, \quad \bar{c}^{2}=(\gamma-1)\left[H-\frac{1}{2} \bar{u}^{2}\right],
$$

giving for the eigenvalues and right eigenvectors

$$
\begin{gathered}
\bar{\lambda}^{1}=\bar{u}, \quad \bar{\lambda}^{2}=\bar{u}+\bar{c}, \quad \bar{\lambda}^{3}=\bar{u}-\bar{c}, \\
\overrightarrow{\mathbf{r}}^{1}=\left(\begin{array}{c}
1 \\
\bar{u} \\
\frac{1}{2} \bar{u}^{2}
\end{array}\right), \quad \overline{\mathbf{r}}^{2}=\left(\begin{array}{c}
1 \\
\bar{u}+\bar{c} \\
\bar{H}+\overline{u c}
\end{array}\right), \quad \overline{\mathbf{r}}^{3}=\left(\begin{array}{c}
1 \\
\bar{u}-\bar{c} \\
\bar{H}-\overline{u c}
\end{array}\right) .
\end{gathered}
$$

Because of the simpler algebra it is often preferred (e.g. [3]) to compute the discrete wave strengths using the primitive variable $\mathrm{V}=(\rho, u, p)^{\mathrm{T}}$ and the corresponding left eigenvectors $l_{v}^{k}$. Care has then to be taken in a consistent definition of the gradient $\widehat{\mathbf{v}}_{x}$. The crucial relations to be preserved are equations $(6 a, b)$ and hence equation $(7)$ has to be satisfied exactly, written for all components together using the matrices of left eigenvectors $L$ for the conservative and $L_{V}$ for the primitive variable [3]:

$$
\left(\begin{array}{c}
\widehat{\alpha^{1}} \\
\frac{\alpha^{2}}{\alpha^{3}}
\end{array}\right)=L(\overline{\mathbf{Z}}) \widehat{\mathbf{U}_{x}}=L_{V}(\overline{\mathbf{Z}}) \widehat{\mathbf{V}_{x}}=\left[\begin{array}{c}
\frac{1}{\bar{c}^{2}}\left[\bar{c}^{2} \widehat{p_{x}}-\widehat{p_{x}}\right] \\
\frac{1}{2 \bar{c}^{2}}\left[\widehat{p_{x}}+\overline{\rho c} \widehat{u_{x}}\right] \\
\frac{1}{2 \bar{c}^{2}}\left[\widehat{p_{x}}-\overline{\rho c} \widehat{u_{x}}\right]
\end{array}\right]
$$

Hence, the gradient in primitive variables has to be defined as

$$
\widehat{\mathbf{V}_{x}}=L_{\nu}^{-1}(\overline{\mathbf{Z}}) L(\overline{\mathbf{Z}}) \widehat{\mathbf{U}_{x}}=\frac{\partial \mathbf{V}}{\partial \mathbf{U}}(\overline{\mathbf{Z}}) \widehat{\mathbf{U}_{x}}=\frac{\partial \mathbf{V}}{\partial \mathbf{Z}}(\overline{\mathbf{Z}}) \widehat{\mathbf{Z}_{x}}
$$

However, the matrix $\partial \mathbf{V} / \partial \mathbf{Z}$ is not linear in the components of $\mathbf{Z}$, and the consistent derivative is no longer identical to the differences over a cell. Instead, it is given by

$$
\widehat{\mathbf{v}_{x}}=\left(\begin{array}{c}
\widehat{\rho_{x}} \\
\widehat{u_{x}} \\
\widehat{p_{x}}
\end{array}\right)=\left(\begin{array}{c}
2 \sqrt{\bar{\rho} z z_{x}^{1}} \\
\left.\frac{1}{\sqrt{\bar{\rho}}} \widehat{\left[z_{x}^{2}\right.}-\bar{u} \widehat{z_{x}^{1}}\right] \\
\frac{\gamma-1}{\gamma} \sqrt{\bar{\rho}}\left[\widehat{H} \widehat{z_{x}^{1}}-\bar{u} \bar{z}_{x}^{2}+\widehat{z_{x}^{3}}\right]
\end{array}\right)=\frac{1}{\Delta x_{i+\frac{1}{2}}}\left[\begin{array}{c}
\rho_{i+1}-\rho_{i} \\
\frac{\tilde{\rho}}{\bar{\rho}}\left(u_{i+1}-u_{i}\right) \\
p_{i+1}-p_{i}
\end{array}\right],
$$

where $\tilde{\rho}=\sqrt{\boldsymbol{\rho}_{i} \rho_{i+1}}$ is the well-known Roe averaged density $[1,3]$, while $\bar{\rho}=\rho(\overline{\mathbf{Z}})=\left[\left(\sqrt{\rho_{i}}+\sqrt{\rho_{i+1}}\right) / 2\right]^{2}$ is the consistent definition introduced before. Indeed, substituting $\widehat{\rho_{x}}, \widehat{u_{x}}$ and $p_{x}$ in the consistent expressions (9), one sees that the consistent averaged density $\bar{\rho}$ cancels, and one obtains the usual expressions needing only the definition of $\tilde{\rho}$. The use of the consistent formulation is however necessary if one wishes to generalize the expressions in two space dimensions.

Equations $(6 \mathrm{a}, \mathrm{b})$ have the important property that whenever $\mathbf{U}_{i}, \mathbf{U}_{i+1}$ are such that they can be connected by a single shock wave or a single contact discontinuity, only one nonvanishing term in the expansion remains, and equation (6b) reduces to the Rankine-Hugoniot jump relations, with $\overline{\lambda^{k}}$ the speed of the discontinuity, thus ensuring uniform validity of the linearization both in smooth flow and near discontinuities.

\subsection{Scalar distribution scheme}

In the third step, a scalar upwind distribution scheme is applied to each part of the decomposed flux residual given by equations $(6 \mathrm{a}, \mathrm{b})$, depending on the orientation of the corresponding speed $\bar{\lambda} \vec{I}_{x}$. For example, in the classical first-order upwind scheme, the terms $\bar{\lambda}^{k} \widehat{\alpha^{k}} \overline{\mathbf{r}}^{k}$ corresponding to 
$\overline{\lambda^{k}} \geqslant 0$ are used to update $\mathbf{U}_{i+1}$, while the parts corresponding to $\overline{\lambda^{k}} \leqslant 0$ are used to update $\mathbf{U}_{i}$. Other schemes, like the Lax-Wendroff or Fromm schemes, can be recovered by selecting other distribution coefficients [1]. The key observation is that the problem is reduced to the design of accurate and oscillation-free distribution schemes for a scalar advection equation. Because of the conservative linearization, the fluxes at the meshpoints are never needed. It is sufficient to compute the linearized eigenvectors and speeds, and the wave strengths over a cell. Hence, the standard first-order finite volume scheme can be written in the following alternative formulation:

1. Compute for each cell the conservative residual

$$
\mathbf{R}_{i+\frac{1}{2}}=\sum_{k=1}^{3} \mathbf{R}_{i+\frac{1}{2}}^{k}=-\Delta x_{i+\frac{1}{2}} \sum_{k=1}^{3} \bar{\lambda}^{k} \widehat{\alpha^{k}} \tilde{\mathbf{r}}^{k}
$$

2. Send for each cell the contribution $\left(\Delta t / \Delta x_{i}\right) \mathbf{R}_{i+\frac{1}{2}}^{k}$ to node $i$ if $\bar{\lambda}^{k} \geqslant 0$ and $\left(\Delta t / \Delta x_{i+1}\right) \mathbf{R}_{i+\frac{1}{2}}^{k}$ to node $i+1$ if $\bar{\lambda}^{k}<0$, where $\Delta x_{i}$ is the length of the median dual cell around meshpoint $i$, given by $\Delta x_{i}=\frac{1}{2}\left(\Delta x_{i-\frac{1}{2}}+\Delta x_{i+\frac{1}{2}}\right)$.

Hence, the scheme sends contributions from two adjacent cells to a given node, and the general update identical to the standard first-order finite volume scheme can be written as

$$
\mathbf{U}_{i}^{n+1}=\mathbf{U}_{i}^{n}+\frac{\Delta t}{\Delta x} \sum_{i=1}^{3}\left(\left[\beta_{i+\frac{1}{2}}^{i}\right]^{k} \mathbf{R}_{i+\frac{1}{2}}^{k}+\left[\beta_{i-\frac{1}{2}}^{i}\right]^{k} \mathbf{R}_{i-\frac{1}{2}}^{k}\right),
$$

where the downstream distribution coefficients $\beta$ have been defined for each meshpoint of a cell, given by

$$
\left[\beta_{i+\frac{1}{2}}^{i}\right]^{k}=0, \quad\left[\beta_{i+\frac{1}{2}}^{i+1}\right]^{k}=1 \text { for } \bar{\lambda}^{k} \geqslant 0
$$

or

$$
\left[\beta_{i+\frac{1}{2}}^{i}\right]^{k}=1,\left[\beta_{i+\frac{1}{2}}^{i+1}\right]^{k}=0 \text { for } \bar{\lambda}^{k}<0
$$

\section{MULTIDIMENSIONAL GENERALIZATION}

Consider now the 2-D Euler equations in conservation form:

$$
\mathbf{U}_{t}+\mathbf{F}_{x}+\mathbf{G}_{y}=0
$$

where $\mathbf{F}$ and $\mathbf{G}$ are the Cartesian components of the flux vector in 2-D, with Jacobians $A(\mathbf{U})=\mathbf{F}_{\mathbf{U}}$ and $B(\mathbf{U})=\mathbf{G}_{\mathbf{U}}$. Each of the steps used in the formulation of Roe's classical scheme as described in the previous section generalizes in a quite straightforward way.

\subsection{Multidimensional eigenvector decomposition}

In two space dimensions, an eigenvector decomposition of the flux divergence $\mathbf{F}_{x}+\mathbf{G}_{y}$ is needed, starting from a decomposition of the space gradient of the conservative variable. Different approaches have been formulated [4,5], but because of limited space, we restrict ourselves to a brief discussion of Roe's simple wave decomposition [4]. It is based on a superposition of simple wave solutions, assuming linearized flow, and is defined by

$$
\mathrm{U}^{k}(x, y, t)=\alpha^{k} \mathbf{r}^{k} \cdot\left(x n_{x}^{k}+y n_{y}^{k}-\lambda_{n}^{k} t\right),
$$

where $\alpha^{k}$ is again the strength of the wave, $n_{x}^{k}=\cos \theta^{k}$ and $n_{y}^{k}=\sin \theta^{k}$ are the components of the unit vector $\mathbf{i}^{k}$ in the propagation direction $\theta^{k}$ and $r^{k}$ is a right eigenvector of the Jacobian $A n_{x}^{k}+B n_{y}^{k}$ with the corresponding eigenvalue $\lambda_{n}^{k}$. Each simple wave is associated with a scalar advection equation in 2-D with advection speed vector $\vec{\lambda}_{n}^{k}=\lambda_{n}^{k} \overrightarrow{\mathrm{i}}_{n}^{k}$. Hence, the 2-D generalization of equation $(\mathrm{la}, \mathrm{b})$ is given by

$$
\vec{\nabla} \mathbf{U}=\sum_{k} \alpha^{k} \mathbf{r}^{k} \tilde{n}^{k}, \quad \mathbf{F}_{x}+\mathbf{G}_{y}=\sum_{k} \lambda_{n}^{k} \alpha^{k} \mathbf{r}^{k}
$$

Matching the local gradient with a superposition of simple waves of the above type defines the decomposition, precisely as in 1-D, equation (1a). In 1-D there is only 1 degree-of-freedom per 
wave, namely its strength $\alpha^{k}$, and the basis consists of 3 independent eigenvectors, while the space derivative $\mathbf{U}_{x}$ has 3 components. Therefore, equation (1a) leads to a system of 3 equations for the 3 strengths, and the decomposition is unique.

In two space dimensions, the matching equation (2a) provides 8 equations, 4 for the $x$-derivative and 4 for the $y$-derivative. On the other hand, each simple wave has two parameters, its strength $\alpha^{k}$ and its normal $\tilde{\mathbf{n}}^{k}$. Therefore, if all angles and strengths are left free, only 4 waves can be retained in the summation, e.g. corresponding to 1 entropy, 1 shear and 2 acoustic waves. However, the algebra which comes out of this model is too complex and other models have been proposed, adding more waves by specifiying the additional degrees of freedom based on physical arguments. For example, simple algebra results if one considers the following 6-wave model:

(1) One entropy wave with unknown direction and intensity: 2 unknowns.

(2) A shear wave with unknown strength traveling in a specified direction.

(3) Four mutually perpendicular acoustic waves, contributing 5 unknowns: 1 direction and 4 strengths.

The choice of the direction of the shear wave leaves some room for discussion. Roe proposed the direction perpendicular to the streamlines (model B). A better choice (model C) [6], is the direction of the pressure gradient. In any case, closed-form expressions are obtained for the angles and wave strengths, just like in 1-D for the wave strengths.

\subsection{Conservative linearization in $2-D[8]$}

The generalization of the continuous, piecewise linear representation of the unknowns requires a discretization on triangles with unknowns defined at the vertices, precisely as in the finite element method using linear elements. Discrete gradients are again defined as exact integrals over a triangle $T$ with surface $S_{T}$, assuming linear variation of a particular variable $\mathbf{Q}$ to be chosen below:

$$
\widehat{\mathbf{v} U}=\frac{1}{S_{T}} \iint_{S_{T}} \vec{\nabla} \mathbf{U}(\mathbf{Q}) \mathrm{d} S, \quad \widehat{\mathbf{F}_{x}}=\frac{1}{S_{T}} \iint_{S_{T}} \mathbf{F}_{x}(\mathbf{Q}) \mathrm{d} S, \quad \widehat{\mathbf{G}_{y}}=\frac{1}{S_{T}} \iint_{S_{T}} \mathbf{G}_{y}(\mathbf{Q}) \mathrm{d} S .
$$

Since all integrations are exact, the contour integral of the fluxes over a triangle is given by

$$
\oint_{S_{T}} \overrightarrow{\mathbf{F}^{n} \mathrm{n}} \mathrm{d} l=\iint_{S_{T}}\left(\mathbf{F}_{x}+\mathbf{G}_{y}\right) \mathrm{d} S=S_{T}\left[\widehat{\mathbf{F}_{x}}+\widehat{\mathbf{G}_{y}}\right] .
$$

Hence, for any choice of the underlying variable $\mathbf{Q}$ having linear variation over the triangle, the flux contour integrals over the triangles will telescope over the domain, provided that the integrations in equations $(16 \mathrm{a}-\mathrm{c})$ are exact, thus generalizing the telescoping property of the flux differences in 1-D. Moreover, for the same reasons and following exactly the same steps as in 1-D, choosing the parameter vector $\mathbf{Z}=\left(z^{1}, z^{2}, z^{3}, z^{4}\right)^{\mathrm{T}}=\sqrt{\rho}(1, u, v, H)^{\mathrm{T}}$ leads to an exact linearization of the form

$$
\widehat{\mathbf{F}_{x}}+\widehat{\mathbf{G}_{y}}=A(\overline{\mathbf{Z}}) \widehat{\mathbf{U}_{x}}+B(\overline{\mathbf{Z}}) \widehat{\mathbf{U}_{y}},
$$

where the linearized Jacobians are again the analytical expressions $A=\mathbf{F}_{\mathrm{U}}$ and $B=\mathbf{G}_{\mathrm{U}}$, evaluated at the state $\overline{\mathbf{Z}}$, which is now the arithmetic average of the values at the vertices of the triangle:

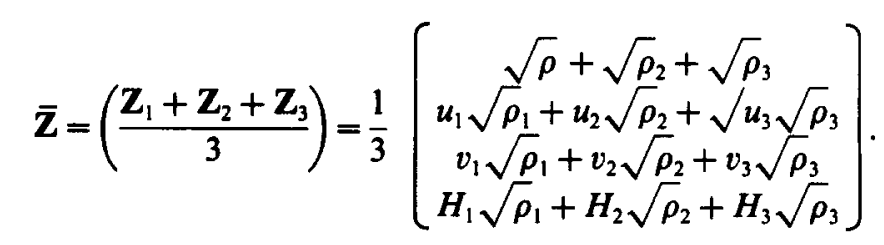

Once a conservative linearization in the form of equation (18) has been achieved, the conservative residual is easily decomposed using the 6-wave model [equation (15b)], giving the 2-D generalization of equation (11): 


$$
\mathbf{R}_{T}=-\oint_{S_{T}} \overrightarrow{\mathbf{F}^{\dagger}} \mathbf{d} l=-S_{T}\left(\widehat{\mathbf{F}_{x}}+\widehat{\mathbf{G}_{y}}\right)=-S_{T} \sum_{k=1}^{6} \bar{\lambda}^{k} \hat{\alpha}^{k} \overline{\mathbf{r}}^{k}=\sum_{k=1}^{6} \mathbf{R}_{T}^{k},
$$

where the linearized eigenvectors and eigenvalues are again the analytical expressions resulting from the model, evaluated in the state $\overline{\mathbf{Z}}$. For example, in the state $\overline{\mathbf{Z}}$, one has

$$
\left[\begin{array}{c}
\bar{\rho} \\
\bar{u} \\
\bar{v} \\
\bar{H}
\end{array}\right]=\left[\begin{array}{c}
\frac{\left(\sqrt{\rho_{1}}+\sqrt{\rho_{2}}+\sqrt{\rho_{3}}\right)^{2}}{9} \\
\frac{u_{1} \sqrt{\rho_{1}}+u_{2}{\sqrt{\rho_{2}}}+u_{3} \sqrt{\rho_{3}}}{\sqrt{\rho_{1}}+\sqrt{\rho_{2}}+\sqrt{\rho_{3}}} \\
\frac{v_{1} \sqrt{\rho_{1}}+v_{2}{\sqrt{\rho_{2}}}_{2}+v_{3} \sqrt{\rho_{3}}}{\sqrt{\rho_{1}}+\sqrt{\rho_{2}}+\sqrt{\rho_{3}}} \\
\frac{H_{1} \sqrt{\rho_{1}}+H_{2}{\sqrt{\rho_{2}}}_{3} H_{3} \sqrt{\rho_{3}}}{\sqrt{\rho_{1}}+\sqrt{\rho_{2}}+\sqrt{\rho_{3}}}
\end{array}\right] .
$$

Again as in 1-D, it is preferred to compute the wave strengths and propagation angles using the matching equation in the primitive variables $\mathrm{V}=(\rho, u, v, p)^{\mathrm{T}}$, because the algebra involved is much simpler [4]. Thus, the strengths and angles are computed by solving the following system instead of using equation (15a):

$$
\widehat{\mathbf{V}} \widehat{\mathbf{V}}=\sum_{1}^{6} \widehat{\alpha^{k}} \tilde{\mathbf{n}}^{k} \tilde{\mathbf{r}}_{V}^{k},
$$

where $\overline{\mathrm{i}}_{V}^{k}$ are the right eigenvectors in primitive variables, evaluated in the state $\overline{\mathbf{Z}}$. The consistent expression equivalent to equation (10), to be used in the LHS, is given by Ref. [9]:

$$
\vec{\nabla} \mathbf{V}=\frac{\partial \mathbf{V}}{\partial \mathbf{Z}}(\mathbf{Z}) \overrightarrow{\mathbf{\nabla} Z}=\left[\begin{array}{c}
\hat{\nabla} \hat{\rho} \\
\vec{\nabla} u \\
\hat{\nabla} v \\
\hat{\nabla} p
\end{array}\right]=\left[\begin{array}{c}
2 \sqrt{\bar{\rho}} \vec{\nabla} z^{1} \\
\frac{1}{\sqrt{\bar{\rho}}}\left[\vec{\nabla} z^{2}-\vec{u} \vec{\nabla} z^{1}\right] \\
\frac{1}{\sqrt{\bar{\rho}}}\left[\vec{\nabla} z^{3}-\bar{v} \vec{\nabla} z^{1}\right] \\
\frac{\gamma-1}{\gamma} \sqrt{\bar{\rho}}\left[\bar{H} \vec{\nabla} z^{1}-\bar{u} \vec{\nabla} z^{2}-\bar{v} \vec{\nabla} z^{3}+\vec{\nabla} z^{4}\right]
\end{array}\right]
$$

Because of our assumptions, the gradients of the parameter vector components in the RHS are just constants, easily evaluated for a given triangle.

\subsection{Scalar distribution schemes in 2-D}

Since each part $\bar{\lambda}^{k} \widehat{\alpha^{k}} \overline{\mathbf{r}}^{k}$ of the decomposed residual is associated with a particular advection speed vector $\overline{\bar{\lambda}^{k}}$ with known orientation in the 2-D plane, the downwind distribution schemes discussed in section 1.3 generalize naturally as follows $[7,9]$ :

1. Compute for each triangular cell $T$ the conservative residual

$$
\mathbf{R}_{T}=\sum_{k=1}^{6} \mathbf{R}_{T}^{k}=-S_{T} \sum_{k=1}^{6} \bar{\lambda}^{k} \widehat{\alpha}^{k} \overline{\mathbf{r}}^{k} \text {. }
$$

2. Send for each cell and for each wave $k$ the contribution $\left(\Delta t / S_{p}\right)\left[\beta_{T}^{p}\right]^{k} \mathbf{R}_{T}^{k}$ to the vertices $p$, where the coefficients $\left[\beta_{T}^{p}\right]^{k}$ for the three vertices sum up to one for a given wave, while the area $S_{p}$ is the surface of the median dual cell around meshpoint $p$, given as one-third of the area of the triangles surrounding $p$.

The distribution coefficients $\left[\beta_{T}^{p}\right]^{k}$ are again determined in an upwind manner, depending on the orientation of the advection speed vector $\overline{\bar{\lambda}^{k}}$. For example, if the triangle has a single inflow side for a given advection speed vector $\vec{\lambda}^{k}$, there is only one downstream vertex and the corresponding 
coefficient $\left[\beta_{T}^{p}\right]^{k}$ can be chosen as unity, such that the entire residual $\mathbf{R}_{T}^{k}$ will be sent to that downstream vertex, exactly as in the 1-D case discussed before.

Similar to the 1-D case, the scheme sends contributions from all cells surrounding a given node, and the updating formula generalizing equation (12) for a given node $i$ can be written as

$$
\mathbf{U}_{i}^{n+1}=\mathbf{U}_{i}^{n}+\frac{\Delta t}{S_{i}} \sum_{k=1}^{6} \sum_{\tau}\left[\beta_{T}^{i}\right]^{k} \mathbf{R}_{T}^{k}
$$

where the second summation extends over all triangles with common vertex $i$.

The key observation is again that the problem is reduced to the construction of accurate and monotonic schemes for a scalar advection equation in 2-D. A general theory for constructing such schemes has been developed [7,9], based on the concepts of positivity and linearity preservation. Positivity imposes a maximum principle on the discrete solution, thus providing stability and monotonic discontinuity capturing, while linearity preservation guarantees that an exact steady-state solution is preserved by the scheme whenever such an exact solution has linear variation in space. Precisely as in 1-D with the TVD schemes, nonlinear schemes are needed if one wishes to combine both properties, even for a linear advection equation.

Another remarkable property is that scheme (24) is conservative, while no flux evaluations are needed: summing up equation (24) for all meshpoints one obtains in the RHS a summation of all the cell residuals which telescope because of the conservative linearization (20), thus leaving only the boundary contributions of the fluxes.

\section{COMPUTATIONAL EXAMPLES AND CONCLUSIONS}

The first example, shown in Fig. 1, is the reflection of an oblique shock on a flat plate with an incoming Mach number of 2.9 and an incident shock angle of $29^{\circ}$. The second test case, shown in Fig. 2, is a supersonic flow in a channel with bump of $2 \%$ thickness, with an incoming Mach number of 1.4. In both figures, the solution is presented in the form of iso-Mach lines superimposed on the grid. For both computations a nonlinear positive and linearity-preserving distribution

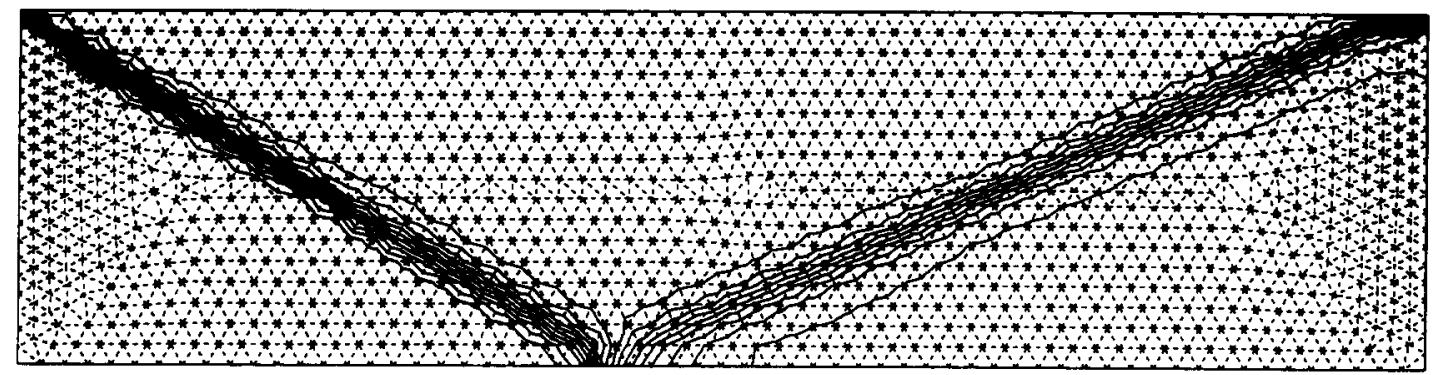

Fig. 1. Oblique shock reflection with an upstream Mach number of 2.9 and a shock angle of $29^{\circ}$. Iso-Mach lines with increment 0.05 , superimposed on the mesh (1235 vertices). Wave model $\mathrm{C}, \mathrm{NN}$ scheme.

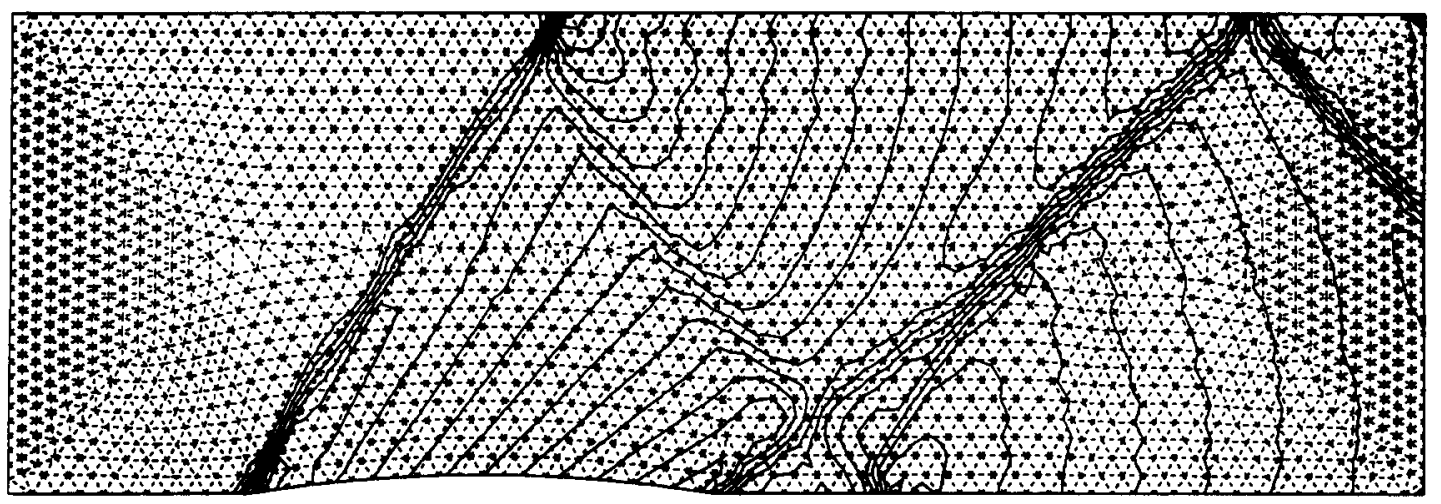

Fig. 2. Supersonic channel flow with an inlet Mach number of 1.4. Iso/Mach lines with increment 0.05 , superimposed on the mesh (1977 vertices). Wave model C, NN scheme. 
scheme (NN scheme, [7]) with model $\mathrm{C}$ has been used. Shock capturing within a layer of about 2 cells is obtained, with little influence by the grid.

These results are numerical evidence that the multidimensional generalization of Roe's scheme as described in this paper represents a viable alternative for the well-established dimensionally split (or locally 1-D) TVD upwind solvers, in particular on unstructured grids composed of triangles. Nevertheless, the results obtained to date should only be viewed as preliminary, especially for subsonic and transonic flow. The most critical item requiring additional refining is probably the multidimensional eigenvector decomposition (section 2.2), since only one of the models considered so far (6-wave model, C) gave satisfactory results for all the test cases considered [9].

Acknowledgement - The work at VKI was supported by the Commision of the European community under Contract AERO-0003-C in Area 5 (Aeronautics) of the BRITE/EURAM Program (1989-1993).

\section{REFERENCES}

1. P. L. Roe, The use of the Riemann problem in finite differences. In Proceedings of the 7th International Conference on Numerical Methods in Fluid Dynamics (Edited by W. C. Reynolds and R. W. MacCormack). Springer-Verlag, Berlin (1981).

2. P. L. Roe, Approximate Riemann solvers, parameter vectors, and difference schemes. J. Comput. Phys. 43,357 (1981).

3. P. L. Roe, Characteristics based schemes for the Euler equations. A. Rev. Fluid Mech. 18, 337 (1986).

4. P. L. Roe, Discrete models for the numerical analysis of time-dependent multidimensional gasdynamics. J. Comput. Phys. 63, 458 (1986).

5. H. Deconinck, Ch. Hirsch and J. Peuteman, Characteristic decomposition methods for the multidimensional Euler equations. In Lecture Notes in Physics, Vol. 264. Springer-Verlag, Berlin (1986).

6. P. De Palma, H. Deconinck and R. Struijs, Investigation of Roe's 2D wave models for the Euler equations. Report VKI TN172 (1990).

7. R. Struijs, H. Deconinck and P. L. Roe, Fluctuation splitting schemes for the 2D Euler equations. VKI Lecture Series 1991-01 (1991).

8. P. L. Roe, R. Struijs, and H. Deconinck, A conservative linearization of the multidimensional Euler equations. $J$. Comput. Phys. (1993).

9. R. Struijs, H. Deconinck, P. De Palma, P. L. Roe and K. G. Powell, Progress on multidimensional upwind Euler solvers for unstructured grids. AIAA Paper 91-1550 (1991). 